\title{
INTERCHANGE HETEROZYGOSITY AND SELECTION IN RYE
}

\author{
R. J. BAILEY and H. REES \\ Department of Agricultural Botany, U.C.W., Aberystwyth \\ and \\ M. A. ADENA \\ Department of Population Biology, Research School of Biological Sciences, \\ Australian Notional University, Canberra
}

Received 14.ix.77

\begin{abstract}
SUMmary
The break-points for an interchange in rye are such that all three combinations, the normal structural homozygote, the interchange heterozygote and homozygote were readily identifiable at both mitosis and meiosis. Most of the interchange homozygotes are inviable. In populations subjected to increasing selection pressures imposed by high sowing density the proportion of interchange heterozygotes among survivors increased. In terms of survival (seed to adult viability) the interchange heterozygotes at high sowing densities have a 102 per cent advantage over the normal homozygotes, more than sufficient to generate a stable polymorphism for the interchange. The general requirements for the maintenance of interchange are considered and analysed in relation to the degree of heterozygote advantage, the effects of the structural change upon fertility and to the breeding systems of natural populations.
\end{abstract}

\section{INTRODUCTION}

THE fertility of individuals heterozygous for interchanges (reciprocal translocations) is invariably reduced, often drastically, in comparison with normal structural homozygotes. Even so, interchanges are found floating in surprisingly high frequencies among outbreeding populations in plant species (Stebbins, 1963) and, occasionally, fixed as permanent heterozygotes, e.g. in Oenothera (Darlington, 1931; Cleland, 1949) and Rhoeo (Sax, 1931). Interchanges have been found floating also in animal populations, e.g. in Periplaneta americana (John and Lewis, 1958) but in animals, in general, they are rare (White, 1973). To account for the accumulation and maintenance of interchanges in high frequencies it is clear that their contribution to fitness must more than compensate for the infertility of heterozygotes. The evidence from Campanula (Darlington and Gairdner, 1938), Periplaneta (John and Lewis, 1958), and rye (Rees, 1961) indicates that a major factor is the superior fitness of the heterozygotes in the face of selection. In order to detect and analyse the effects of selection on an experimental basis the ideal requirements are that all three chromosome combinations, the interchange homozygote, the interchange heterozygote, and, as well, the normal, wild type structural homozygote be readily distinguished at mitosis and meiosis. We may then compare the response of plants carrying the different chromosome combinations to the selection pressures applied. An interchange in rye fulfils these requirements and the following is an account of this interchange with particular emphasis upon its effect among populations exposed to severe selection pressure imposed by high sowing density.

$$
41 / 1-\mathrm{A}
$$




\section{MAterial AND METHODS}

(i) The interchange stocks

The interchange was found in 1971 in a population derived initially from crosses between Secale cereale and $S$. vavilovi (see Jones and Rees, 1967). It appeared in a single plant which was heterozygous for the interchange. The population had been screened over a number of previous generations and since only this one plant was found to carry the interchange it is probable that the interchange arose spontaneously in this individual.

The one plant was allowed to open-pollinate in the presence of normal structural homozygotes from the same population. Its progeny was grown in isolation and allowed to open-pollinate. A further generation of open pollination provided seeds for experiment 1 (1973) and one more generation gave seeds for the second experiment (1974). The seeds obtained from these open-pollinated populations were too few in themselves for carrying out the experiments. For this reason they were mixed thoroughly with seeds from open-pollinated normal plants (structural homozygotes) derived from the same initial population.

Although the seeds used for the experiments were from open-pollinated plants they were by no means exclusively products of cross-pollination. Plants from these populations are largely self-pollinating. They set ample seed when "foreign" pollen is excluded. Using $B$ chromosomes as markers, the degree of self-pollination was estimated to be 40 per cent. The element of inbreeding needs to be emphasised. It is relevant to the consideration of the role of interchanges within populations.

\section{(ii) Density experiments}

Seeds were sown, in John Innes No. 2 potting compost, in "multipot" plastic trays, each pot having a diameter of only $5 \mathrm{~cm}$ and a depth of $6.4 \mathrm{~cm}$. Seeds were sown in pots at different densities with the object of adjusting the degree of competition among individuals.

Experiment 1. Seeds were planted $1 \mathrm{~cm}$ deep at densities of 5, 10, 25, 50, 100 and 150 per pot in September 1972, kept in a heated greenhouse for 3 weeks and then transferred to an unheated greenhouse. There were three replicates, each of three pots, at each density.

Experiment 2. Seven replicates were sown, each made up of the following; one pot with 150 seeds, one pot with 50 , three pots with 10 , three pots with 5 and 15 pots with 1 seed. Sowing was on 4th February 1974 and seedlings were moved from a heated to an unheated greenhouse after 3 weeks.

The number of plants surviving to maturity (flowering) was scored in each pot. Flowering heads were fixed in Carnoy's solution at random from populations at each density for classifying the plants at meiosis with respect to interchange. Progenies from selfs and crosses were classified by scoring Feulgen stained metaphases in seedling root meristems.

\section{StRUCTURE AND INHERITANCE}

The metaphase in fig. 1 is from an interchange heterozygote. One of the chromosomes $(L)$ is exceptionally long, the $S$ chromosome exceptionally short. They result from a markedly asymmetrical interchange involving the nucleolus organising chromosome. The asymmetry makes easy the 
classification of all three interchange classes both at mitosis and meiosis (fig. 2).

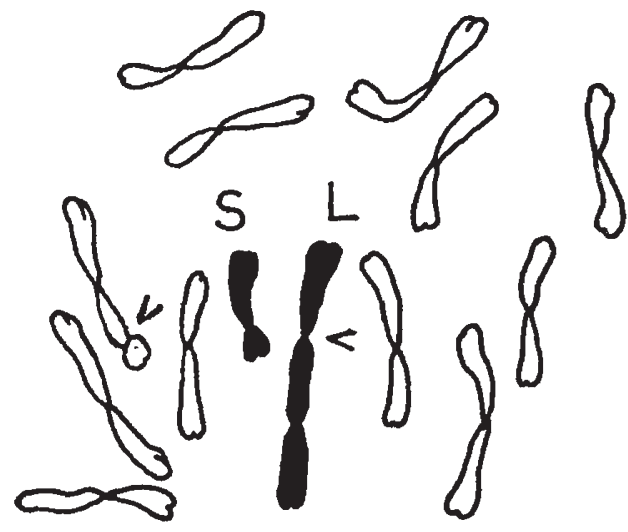

FIG. 1.-Metaphase of mitosis in the $A C$ interchange heterozygote. Arrows mark the constrictions at nucleolus organisers. Horizontal bar $=10$ microns.

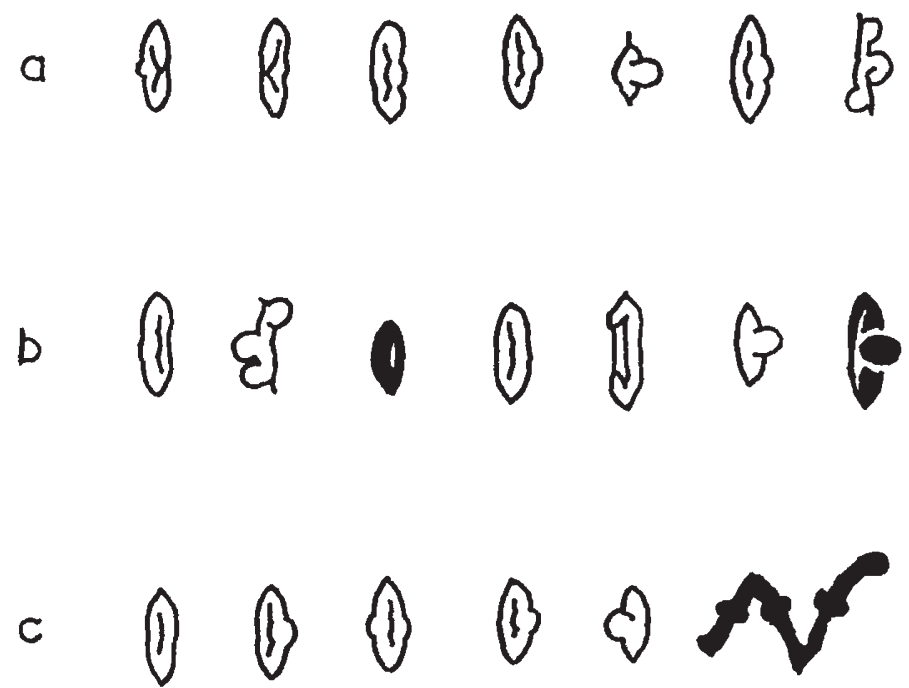

FIG. 2.-Metaphase of meiosis in pollen mother cells of $(a)$ the normal structural homozygote, $(b)$ the interchange homozygote with one small and one large bivalent (in black), (c) the interchange heterozygote with a chain of four (in black).

The progenies of crosses between interchange heterozygotes $(A C) \times$ normal homozygotes $(A A)$ consist of interchange heterozygotes $(A C)$ and normal homozygotes $(A A)$ in the expected $1: 1$ ratio, e.g. $29: 34$, showing 
that the gametes carrying the interchange chromosomes function efficiently and that the $A C$ zygotes, heterozygous for interchange, develop into viable seeds. In contrast, only a small proportion (about 12 per cent) of the $C C$ zygotes, homozygous for interchange, develop into mature seeds. The ratios of $C C$ to $A C$ and $A A$ seeds among progenies of $A C \times A C$ crosses and $A C$ selfs respectively were $3: 33$ and $0: 43$, highly significant departures from the expected $1: 3$. That the scarcity of $C C$ is due to failure of seed development rather than failure to germinate was established from the fact that the percentage germination among seeds from $A C \times A C$ crosses or selfs is the same as for seeds from $A A \times A A$ and $A A \times A C$ crosses.

\section{Competition AND SELection}

(i) Survival in relation to sowing density

It will be recalled that the pots used in the density experiments are small and as the number of plants per pot increased the competition for light and nutrients became increasingly severe. At high densities only 15 to 17 per cent of plants survive and develop to maturity, i.e. to flowering, as compared with 44 to 93 per cent at low densities (tables 1 and 2). While the

\section{TABLE 1}

The distribution of interchange classes among survivors in populations sown at different densities in experiment 1. Data pooled over replicates

$\begin{array}{cccccc}\begin{array}{c}\text { Sowing } \\ \text { density }\end{array} & \begin{array}{c}\text { Per cent } \\ \text { mortality }\end{array} & \begin{array}{c}\text { Interchange } \\ \text { homozygotes }\end{array} & \begin{array}{c}\text { Interchange } \\ \text { heterozygotes }\end{array} & \begin{array}{c}\text { Normal } \\ \text { homozygotes }\end{array} & \begin{array}{c}\text { Per cent } \\ \text { heterozygotes }\end{array} \\ 5 & 55 \cdot 6 & 0 & 3 & 11 & 21 \cdot 43 \\ 10 & 53 \cdot 33 & 1 & 4 & 13 & 22 \cdot 22 \\ 25 & 63 \cdot 11 & 1 & 5 & 24 & 16 \cdot 67 \\ 50 & 65 \cdot 33 & 1 & 8 & 21 & 26 \cdot 67 \\ 100 & 83 \cdot 67 & 0 & 9 & 21 & 30 \cdot 00 \\ 150 & 84.67 & 0 & 12 & 18 & 40 \cdot 00\end{array}$

TABLE 2

The distribution of interchange classes among survivors in populations sown at different densities in Experiment 2. Data pooled over replicates

$\begin{array}{cccccc}\begin{array}{c}\text { Sowing } \\ \text { density }\end{array} & \begin{array}{c}\text { Per cent } \\ \text { mortality }\end{array} & \begin{array}{c}\text { Interchange } \\ \text { homozygotes }\end{array} & \begin{array}{c}\text { Interchange } \\ \text { heterozygotes }\end{array} & \begin{array}{c}\text { Normal } \\ \text { homozygotes }\end{array} & \begin{array}{c}\text { Per cent } \\ \text { heterozygotes }\end{array} \\ 1 & 6 \cdot 80 & 4 & 7 & 24 & 20 \cdot 00 \\ 5 & 18 \cdot 57 & 1 & 6 & 28 & 17 \cdot 14 \\ 10 & 30 \cdot 95 & 0 & 7 & 28 & 20 \cdot 00 \\ 50 & 57 \cdot 33 & 0 & 10 & 22 & 31 \cdot 25 \\ 150 & 82 \cdot 29 & 1 & 11 & 22 & 32 \cdot 35\end{array}$

physical circumstances which apply in the experiments are thoroughly unnatural it is important to stress that mortality among grasses and cereals (and hence the selection pressures), even under conditions of cultivation, are often of the order manifested by the present experiments (e.g. Charles, 1961). 
(ii) The proportion of heterozygotes in relation to the sowing density

Tables 1 and 2 also show the distributions of the three interchange classes among the survivors at each density. The scarcity of interchange homozygotes is at once apparent, confirming the results from crossing and selfing. It will be observed that the interchange homozygotes are almost completely inviable at the higher sowing densities of 100 and 150 per pot.

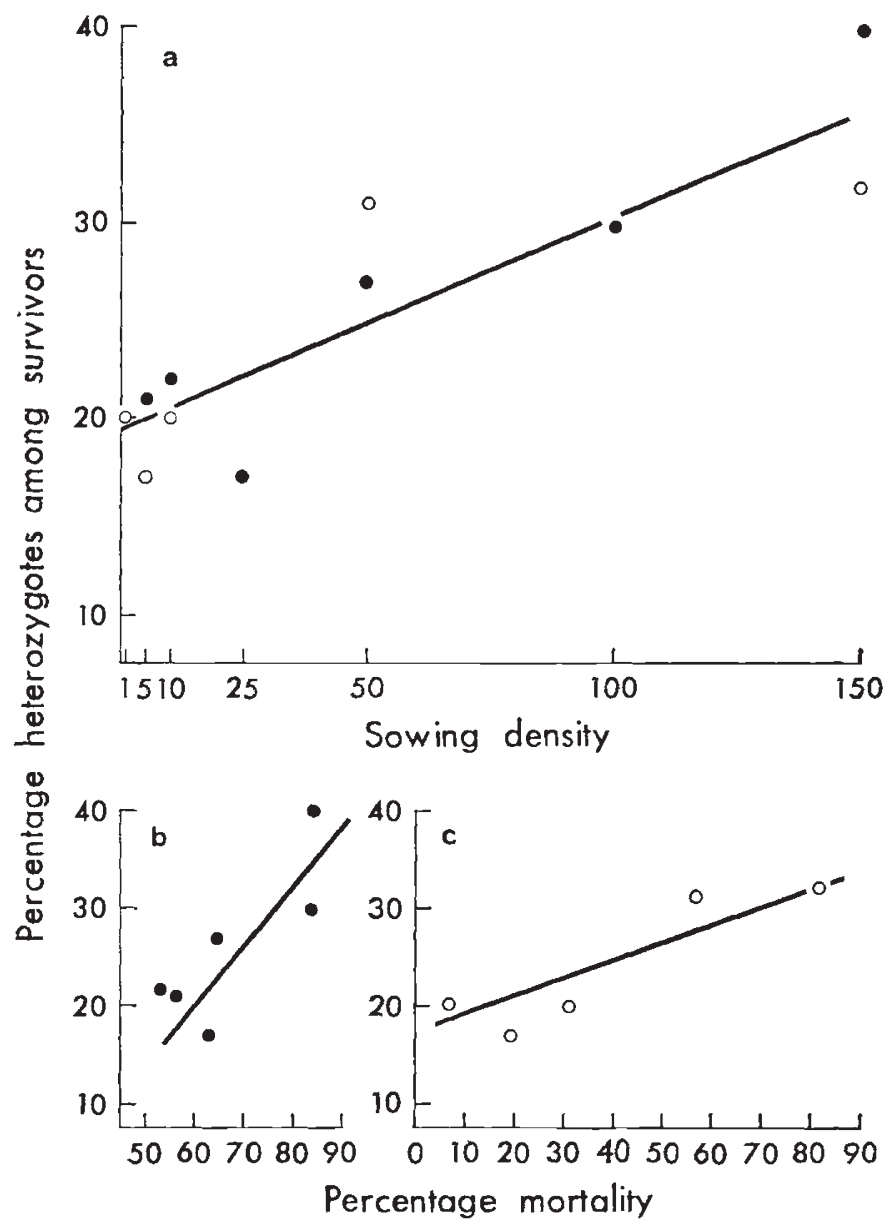

FIG. 3.- (a) The percentage of interchange heterozygotes among survivors of populations sown at different densities. Closed circles, expt. 1; open circles, expt. 2. ( $b$ and $c)$ The percentage of interchange heterozygotes among survivors plotted against the percentage mortality within populations in expts. 1 and 2 respectively.

Tables 1 and 2 and fig. $3 a$ show, among survivors, an increasing proportion of interchange heterozygotes with increasing sowing density. For purposes of analysis we may pool the data not only for replicates as in tables 1 and 2, but also for experiments (table 3 ) because there is no signifi- 


\section{TABLE 3}

The distribution of interchange heterozygotes and of normal plus interchange homozygotes among survivors at different sowing densities. Pooled data from experiments 1 and 2. Per cent heterozygotes in brackets.

$\begin{array}{ccc}\text { Sowing density } & \text { Interchange heterozygotes } & \begin{array}{c}\text { Normal plus } \\ \text { interchange homozygotes }\end{array} \\ 1 & 7(20 \cdot 0) & 28 \\ 5 & 9(18 \cdot 4) & 40 \\ 10 & 11(20 \cdot 8) & 42 \\ 25 & 5(16 \cdot 7) & 25 \\ 50 & 18(29 \cdot 0) & 44 \\ 100 & 9(30 \cdot 0) & 21 \\ 150 & 23(35 \cdot 9) & 41\end{array}$

cant heterogeneity among ratios of heterozygotes to homozygotes between either replicates within densities or between similar densities in the different experiments. A $2 \times j$ analysis of the ratio of heterozygotes to homozygotes gives a total heterogeneity $\chi^{2}$ of $8 \cdot 15$ for 6 degrees of freedom. Of this total $\chi^{2}, 6 \cdot 84$ (for 1 degree of freedom) is due to "trend", the linear increase in the proportion of heterozygotes with increasing density. The increase is highly significant $(P=0.01)$.

We have also analysed the results in tables 1 and 2 by means of a joint regression analysis of variance of the percentage heterozygotes against density. The joint regression, which is linear, is significant at the 1 per cent level, confirming the increase in the proportion of heterozygotes with increasing density. There is no significant heterogeneity between the regressions in the different experiments, from which we conclude also, that the rate of increase of heterozygotes with increasing density is the same for experiments 1 and 2 .

\section{(iii) Heterozygotes and mortality}

The increasing stress upon plant growth and survival with increasing sowing density is directly reflected by the increase in mortality among populations (tables 1 and 2). We can confirm the superiority of heterozygotes with increasing selection pressure at high densities by plotting the percentage heterozygotes against mortality (figs. $3 b$ and $c$ ). The regressions, in both cases, are significant at the 1 per cent level.

\section{(iv) The selection differential}

From the results given in tables 1 and 2 we can estimate the degree of superiority for survival of the interchange heterozygotes relative to the normal homozygotes at the high sowing densities of 100 and 150 . For this purpose we excluded $C C$ homozygotes because it will be recalled, they are very rare ( 1 in 94 ), virtually inviable at these densities. The estimate was derived as follows.

Assume the capacity for the survival of $A C$ and $A A$ to be equal at low sowing densities of 1 to 10 . The ratio of $A C$ to $A A$ among survivors at these low densities will then reflect the ratio of $A C$ to $A A$ seeds sown at the high densities, viz. $27: 104 ; 20.6$ per cent $A C, 79.4$ per cent $A A$. Of the total seeds sown at high densities only 16.5 per cent survive. Of these 32 are 
$A C, 61 A A$. Therefore, of the total seeds sown the survivors comprise5.68 per cent $A C, 10.82$ per cent $A A$. The percentage survival among $A C$ is $28 \cdot 6(5 \cdot 68$ out of $20 \cdot 6)$; among $A A, 14 \cdot 1(10 \cdot 82$ out of $79 \cdot 4)$. The chances of survival among $A C$ are almost exactly twice those of $A A(2 \cdot 02)$. They have an advantage therefore of 102 per cent. In the notation of Hayman (1953) the "seed to adult viability advantage $(v)$ " is $1 \cdot 02$. The advantage, if anything, is underestimated because we have assumed no differential viability at the lower densities. There is evidence to show that interchange heterozygotes, even at low densities are at an advantage (Rees, I961; Bailey et al., 1976).

\section{Prospects for the establishment of the interghange}

(i) The seed output

While there is no doubting the superior fitness of the heterozygotes for survival under severe selection pressures the question remains as to whether, even under these circumstances, the superiority would be sufficient to maintain the interchange within the population from generation to generation, in short to attain a stable polymorphism. In the first place we need to know the number and variety of zygotes produced by $A A$ and $A C$ genotypes in a mixed population. We have to take into account (a) the amount of self-pollination ( 40 per cent), and (b) the degree of disjunction of the interchange association at meiosis in $A C$ heterozygotes. In pollen mother cells the average disjunction frequency at first metaphase, calculated from $10 A C$ plants, is 74 per cent.

From $A A$ plants. Assume the $A A: A C$ ratio to be $1: 1$ and, in the first instance, no differential selection either of $A$ or $C$ gametes or of $A A$ or $A C$ zygotes. By selfing, 40 per cent of zygotes will be $A A$. From $A A \times A A$ and $A A \times A C$ crosses we get $A A$ to $A C$ zygotes in the ratio of $2 \cdot 74$ to 0.74 . The departure from $3: 1$ is explained by the 74 per cent viability of pollen from $A C$ relative to $A A$ plants, corresponding to the disjunction frequency in $A C$ plants. As percentages of the zygotes from crosses they amount to 79 and 21. As percentages of the total zygotes and of seeds from $A A$ they represent 47 and 13 per cent $A A$ and $A C$ respectively. The totals are $87 A A, 13 A C$.

$A C$ plants. Of the 40 per cent zygotes from selfing, 25 per cent will be $A A, 50$ percent $A C$ and 25 per cent $C C$. Of the total zygotes the ratio will be 10 $A A: 20 A C: 10 C C$. From $A C \times A C$ and $A C \times A A$ crosses zygotes will derive from $A$ and $C$ pollen grains in the ratio of $2 \cdot 74$ to 0.74 (reflecting the proportion of $A$ to $C$ pollen). Of the 60 per cent of $A C$ eggs fertilized by crosspollination, therefore, 39 per cent will give $A A$ homozygotes, 50 per cent $A C$ and 11 per cent $C C$. As percentages of the total zygotes the crosses will give $A A: A C: C C$ in the ratio of $23: 30: 7$. From selfs and crosses together we obtain $33 A A: 50 A C: 17 C C$. We must also bear in mind, however, that non-disjunction will cause inviability in female as well as male gametes produced by $A C$ plants. If the degree of non-disjunction in embryo sac mother cells is the same as in pollen mother cells the total zygotes from $100 A C$ eggs will be reduced to $24 A A, 37 A C$ and $13 C C$. Only about 12 per cent of $C C$ zygotes develop into mature seeds. For this reason we expect the seeds set by $A C$ to comprise $24 A A, 37 A C, 1 C C$. Together they amount to 62 per cent of the number of seeds set by $A A$ plants. From $A A$ and $A C$ together the estimated seed output is $111 \mathrm{AA}, 50 \mathrm{AC}, 1 \mathrm{CC}$. 
The estimates above may be contested, in particular, on the grounds that there is no justification for assuming the disjunction frequency of the interchange configuration on the female side in $A C$ plants to be the same (74 per cent) as that observed in pollen mother cells. Cytological verification is impractical. Evidence in support of the estimates was obtained, indirectly, as follows. We set up an experimental population, comprising $10 \mathrm{AA}$ and $10 A C$ plants grown close together but isolated from other rye populations. The average number of seeds set per head by $A C$ plants was $21 \cdot 6 \pm 1 \cdot 6$, 63 per cent of that by $A A$ plants $(34 \cdot 7 \pm 2 \cdot 6)$. The agreement with expectation (62 per cent) is very close.

\section{(ii) The effects of selection}

Selection. We have shown that in the absence of selection favouring $A$ or $C$ gametes, $A A$ or $A C$ zygotes, the progeny of a population comprised of equal numbers of $A A$ and $A C$ plants consisted of $A A$ and $A C$ plants in the ratio of 111 to 50 , along with rare $C C$ homozygotes (see above). In one generation the proportion of heterozygotes has dropped from 50 to 31 per cent. Ignoring $C C$ homozygotes, which make up a negligible fraction of the population by virtue of inviability and infertility, a further generation of breeding reduces the proportion of heterozygotes to 19 per cent. Expressed as proportions of the interchange $C$ chromosomes we have 25 per cent in the initial population, 15 per cent in the first generation progeny, 9 per cent in the second. Computer simulation shows that after seven generations the frequency of interchange $C$ chromosomes would be less than 1 per cent.

While our experiments showed no selection favouring $A$ or $C$ gametes they did reveal, under conditions of high sowing density, a superior capacity for survival (seed to adult viability) of $A C$ heterozygotes relative to $A A$ homozygotes of the order of 102 per cent. Taking into account the lethal nature of $C C$ homozygotes, the disjunction frequency $(0.74)$ and the amount of selfing ( 40 per cent) the theoretical lower limit for the seed to adult advantage if the $C$ interchange is to persist in the population is 69 per cent (see Appendix). 102 per cent advantage is well in excess of this value, more than sufficient to establish a stable polymorphism.

\section{Discussion}

Our experiments showed that heterozygote advantage sufficient to generate a stable polymorphism for interchange $C$ was achieved only under conditions of very severe selection pressure and high mortality. The requirements for maintaining the polymorphism are, of course, the more demanding in this instance because the interchange homozygote, $C C$, is virtually lethal and, as such, a major cause for the loss of the interchange chromosomes. Were the $C C$ homozygotes as viable and fertile as the normal $A A$ homozygotes the survival advantage of the heterozygote necessary to maintain the interchange would be reduced from 69 per cent to 35 per cent (see Appendix).

There are two further possibilities for reducing the degree of heterozygote advantage required to maintain the interchange, namely an increase in the disjunction frequency of the interchange configuration at meiosis in the heterozygote and a reduction in the amount of self-pollination. 
Lawrence (1958) was able to increase the disjunction frequency in rye interchange heterozygotes from 65 to 85 per cent by selection in only three generations. With 85 per cent disjunction the $A C$ heterozygote would require a 47 per cent advantage as compared with 69 (Appendix). Turning to self-pollination, this is a formidable restriction upon the establishment of a stable polymorphism because it promotes the fixation and isolation of homozygotes at the expense of heterozygotes. For this reason a reduction in self-pollination would improve the chances of maintaining the interchange. There is a difficulty, however. In so far as the superiority of the heterozygote stems directly from the superiority of a heterozygous gene complex in a largely homozygous gene background a reduction in selfpollination might well diminish this superiority by reducing the degree of of homozygosity. The consequences, in short, would be conflicting. The conflict might be resolved, however, by generating sufficient homozygosity by crossing among close relatives. Under these circumstances a breeding system that facilitates considerably the maintenance of interchange is one which restricts the matings to normal homozygotes $x$ interchange heterozygotes $(A A \times A C)$. The system precludes the fixation of homozygotes by selfing and precludes the loss of interchange chromosomes where, as with interchange $C$, the homozygote is lethal. In species comprised of unisexual individuals the restriction is not only feasible but commonplace, given that the interchange involves one of the sex chromosomes. Among animals, "aberrant" sex determining mechanisms of the $X Y^{1} Y^{2}$ type are widespread. They result from autosome-sex chromosome interchanges and testify to the superior fitness of the interchange complex over the original normal structural homozygote-albeit a superiority confined and expressed in only one sex. In these cases the matings are, of course, restricted; between the normal homozygotes in one sex and the structural heterozygotes in the other. There are, in fact, cases reported also in dioecious higher plants, in the mistletoes (Barlow and Wiens, 1975; Mechelke, 1976). The males are heterozygous for numerous interchanges, the females homozygous. Of particular interest is that whereas interchanges are common in dioecious species of mistletoe they are virtually absent in the monoecious species (Barlow, personal communication).

Rye, of course, is monoecious and there is no possibility that a system of the mistletoe type could evolve. On the other hand a closely allied system may be envisaged. Rye is normally an outbreeder with a gametophytic incompatibility system preventing inbreeding by selfing. The incompatibility mechanism depends on the expression of alleles at two " incompatibility loci ", $S$ and $Z$ (Lündquist, 1954). The system works as follows. As female parent a plant of genotype $S_{1} S_{2} Z_{1} Z_{2}$ may be fertilised only by pollen carrying alleles which differ either at the $S$ locus or the $Z$. For example, $S_{1} Z_{3}, S_{3} Z_{2}$ pollen grains are compatible, $S_{1} Z_{1}, S_{2} Z_{2}$ are not. Suppose now that by interchange we "link" $S_{1}$ and $Z_{1}$. No interchange homozygotes can arise because of incompatibility and the only possible combinations are interchange heterozygotes and normal homozygotes. Given advantage of the heterozygotes the establishment of the interchange is made easier by eliminating the generation and fixation of interchange homozygotes.

Finally, we need to bear in mind that in considering the fitness of individuals carrying interchanges we have confined our attention exclusively 
to heterozygous advantage arising from genic heterozygosity. We have ignored the possibility of position effect, due to alteration in linkage brought about by interchange. It is not inconceivable that position effects might enhance the fitness both of interchange homozygotes and of heterozygotes. The difficulty is, however, to distinguish a position effect from the many other confounded effects of interchange.

\section{Appendix}

In diploid organisms the population genetics of an interchange system is analogous to the population genetics of two alleles at one locus. Model 1 of Workman and Jain (1966), which is a restatement of a model in Hayman (1953), considers the effects of selection and a mating system consisting of mixed selfing and random mating on the frequencies of two alleles at one locus. Using their notation, this appendix extends their results by giving explicit formulae for the equilibrium karyotype frequencies when either the interchange homozygote is lethal or has viability equal to the normal homozygote.

Let $p, q$ and $r(p+q+r=1)$ be the frequencies of $A A, C C$ and $A C$ plants respectively and let $x, y$ and 1 be their respective viabilities. These viabilities have two components, the relative proportion of gametes produced and the seed to adult viabilities of the different karyotypes at the given sowing density. Let $s$ and $t(s+t=1)$ be the frequencies of selfing and random mating.

(a) Lethal interchange homozygotes. Suppose the interchange homozygote, $C C$, is inviable. Hence $y=0$. Let $f$ be the disjunction frequency for gametes produced by $A C$ plants, and let $v$ be the seed to adult advantage of $A C$ plants over $A A$ plants. The the viability of $A A$ plants relative to $A C$ plants is $x=1 / f(1+v)$. The value of $v$ can be estimated from experiments 1 and 2 described above. The number of $A C$ and $A A$ plants surviving in the low density pots was 27 and 104, while 32 and 61 survived in the high density pots. At the high sowing density the number of $A C$ plants is increased by $(1+v)$ relative to the number of $A A$ plants, that is $32 / 61=$ $(1+v)(27 / 104)$. So the estimate of $v$ is 1.02 . Similarly for a sowing density of 50 plants per pot, where $18 A C$ and $43 A A$ plants survived, the estimate of $v$ is $(18 / 43)(104 / 27)-1=0.61$.

In the case of lethal interchange homozygotes, equation (1) in Hayman (1953) and equations (4a) and (4b) in Workman and Jain (1966) can be solved explicitly to give the stable equilibrium frequency of interchange heterozygotes, $A C$, as $r /(r+f-r f)$ where

$r=1-\left\{(2 x+s x-2)+\left[(2 x+s x-2)^{2}+4(1-s) x(2-x)\right]^{\frac{1}{2}}\right\} /[2(1-s)(2-x)]$

provided $s<2(1-x)$.

This result has been confirmed for several values of $s, f$ and $v$ using a simulation model. This programme was used to produce fig. 4 which shows the frequency of $A C$ plants for 15 generations given the actual values found for rye in this paper, viz. a selfing proportion of $s=0.4$, a disjunction frequency of $f=0.74$ for $A C$ plants and various values of $v$. For these values of $s$ and $f$, a stable polymorphic equilibrium exists if $v$ exceeds $0 \cdot 69$. If the disjunction frequency is as high as 0.85 then $v$ must exceed 0.47 for 


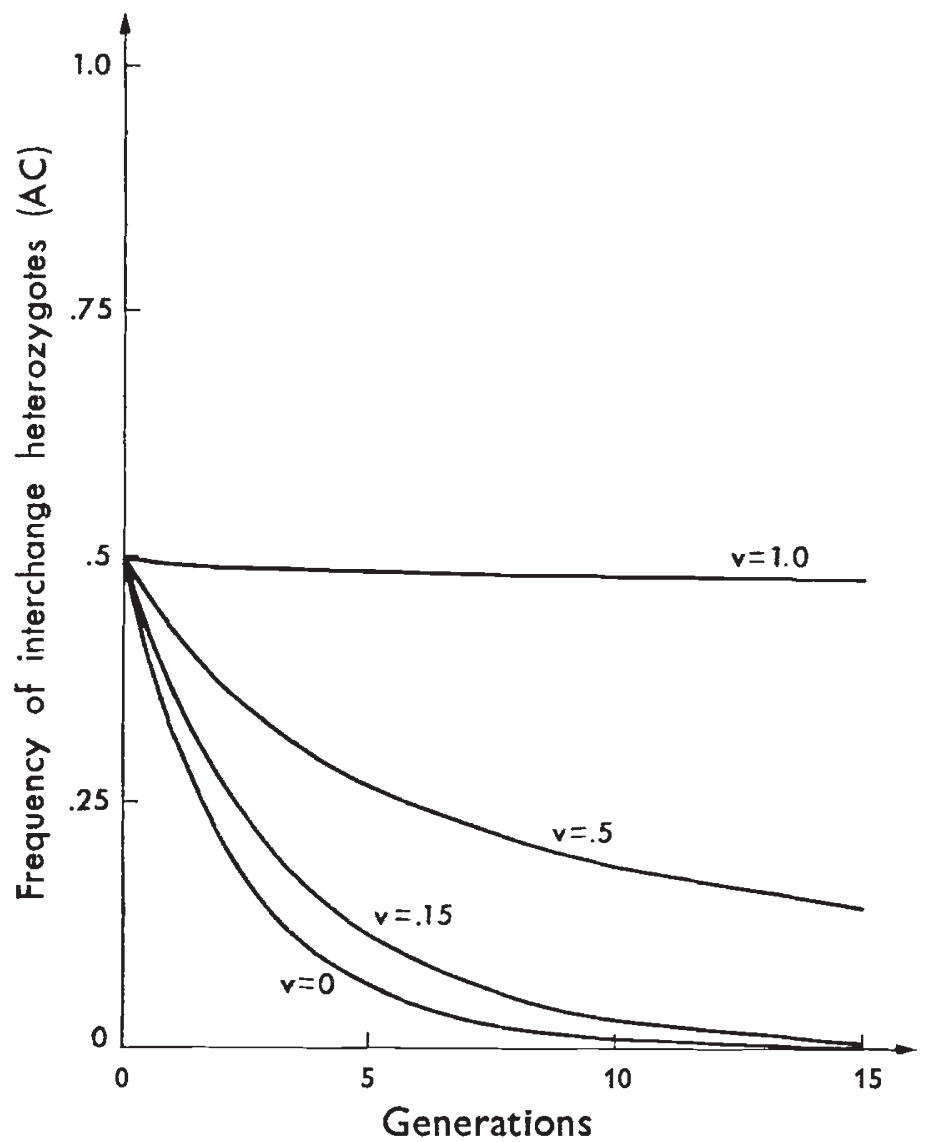

FIG. 4.- The frequency of interchange heterozygotes, $A C$, in 15 generations for various values of $v$, the seed to adult advantage of interchange heterozygotes over normal homozygotes $A A$. The initial $A C$ frequency is 0.5 , the selfing proportion is $s=0.4$ and the disjunction frequency of $A C$ plants is $f=0.74$. The interchange homozygote $C C$ is assumed to be inviable.

the two karyotypes $A A$ and $A C$ to co-exist in a stable equilibrium. For $s=0.4$ and $v=1.02$, as found earlier in this paper, the equilibrium frequency of $A C$ plants is 0.3656 for $f=0.74$, and 0.4945 for $f=0.85$.

(b) Equally viable homozygotes. Suppose now that the two homozygotes, $A A$ and $C C$, are equally viable, that is $x=y$. The same equations in Hayman (1953) and Workman and Jain (1966) can be solved explicitly to show that the stable equilibrium frequency of interchange heterozygotes, $A C$, is given by $\hat{r} /(\hat{r}+f-\hat{r} f)$ where

$$
\hat{r}=1-\left\{(1+x-s x)-\left[(1+x-s x)^{2}-4 s x(1-x)\right]^{\frac{1}{2}}\right\} /[2 s(1-x)]
$$

provided $x<1$.

Therefore the seed to adult viability advantage of $A C$ plants over the homozygotes must exceed $(1 / f-1)$ for both $A$ and $C$ chromosomes to persist in the population. Given a disjunction frequency of $f=0.74$ for $A C$ plants, 
$v$ must exceed 0.35 for a stable polymorphic equilibrium to exist, while for $f=0.85, v$ must exceed $0 \cdot 18$. At a stable polymorphic equilibrium, the frequencies of the two homozygotes, $A A$ and $C C$, are equal (Hayman, 1953).

Acknowledgments.-We are much indebted to Dr A. Durrant of University College of Wales, Aberystwyth, for his advice on statistical methods.

\section{REFERENGES}

BAILEY, R. J., REES, H., AND JONES, L. M. 1976. Interchange heterozygotes versus homozygotes. Heredity, 37, 109-112.

BARLOW, B. A., AND WIENS, D. 1975. Permanent translocation heterozygosity in Viscum hildebrandtii. Engl. and V. engleri Tiegh (Viscaceae) in East Africa. Chromosoma, 53, 265-272.

Charles, A. H. 1961. Differential survival of cultivars of Lolium, Dactylis and Phleum. 7. British Grassland Soc. 16, 69-75.

CLeLAnd, R. E. 1949. Phylogenetic relationships in Oenothera. Proceedings 8 th International Congress of Genetics, Stockholm. Hereditas, suppl. 35, 173-188.

Darlington, C. D. 1931. The cytological theory of inheritance in Oenothera. Four. Gen., 24, 405-474.

DARLington, C. D., AND GAIRdNER, A. E. 1938. The variation system in Campanula persicifolia. Four. Gen., 35, 97-128.

haYman, B. I. 1953. Mixed selfing and random mating when homozygotes are at a disadvantage. Heredity, 7, 185-192.

JoHN, B., AND LEWIS, K. R. 1958. Studies on Periplaneta americana. III. Selection for heterozygosity. Heredity, 12, 185-197.

JONES, R. N., AND REES, H. 1967. Genotypic control of chromosome behaviour in rye. $X I$. The influence of $B$ chromosomes on meiosis. Heredity, 22, 333-347.

LAWRENCE, C. W. 1958. Genotypic control of chromosome behaviour in rye. VI. Selection for disjunction frequency. Heredity, 12, 127-131.

LÜNDQUIST, A. 1954. Studies on self-sterility in rye, Secale cereale L. Hereditas, 40, 278-294. MECHELKE, F. 1976. Sex-correlated complex heterozygosity in Viscum album L. Die Naturwissenschaften, 63, 390.

REES, H. 1961. The Consequence of interchange. Evolution, 15, 145-152.

sax, K. 1931. Chromosome ring formation in Rhoeo discolor. Cytologia, 3, 36-53.

STEBbins, G. L. 1963. Variation and Evolution in Plants. Columbia Univ. Press, New York. white, M. J. D. 1973. Animal Cytology and Evolution, 3rd ed. Cambridge University Press, pp. $271-276$.

WORKMAN, P. L., AND JAIN, S. K. 1966. Zygotic selection under mixed random mating and self-fertilization: Theory and problems of estimation. Genetics, 54, 159-171. 